\title{
COMMENTARIES
}

\section{Using the Clinical Frailty Scale in Allocating Scarce Health Care Resources}

\author{
Kenneth Rockwood, $\mathrm{MD}^{1}$, Olga Theou, $\mathrm{PhD}^{1,2}$ \\ ${ }^{1}$ Division of Geriatric Medicine, Department of Medicine, Faculty of Medicine, Dalhousie University; \\ ${ }^{2}$ School of Physiotherapy, Faculty of Health, Dalhousie University, Halifax, NS
}

https://doi.org/10.5770/cgj.23.463

\begin{abstract}
The key idea behind the Clinical Frailty Scale (CFS) is that, as people age, they are more likely to have things wrong with them. Those things they have wrong (health deficits) can, as they accumulate, erode their ability to do the high order functions which define their overall health. These high order functions include being able to: think and do as they please; look after themselves; interact with other people; and move about without falling. The Clinical Frailty Scale brings that information together in one place. This paper is a guide for people new to the Clinical Frailty Scale. It also introduces an updated version (CFS version 2.0), with revised level names (e.g., "vulnerable" becomes "living with very mild frailty") and minor edits to level descriptions. The key points discussed are that the Clinical Frailty Scale assays the baseline state, it is not widely validated in younger people or those with stable single-system disabilities, and it requires clinical judgement. The Clinical Frailty Scale is now commonly used as a triage tool to make important clinical decisions such as allocating scarce health care resources for COVID-19 management; therefore, it is important that the scale is used appropriately.
\end{abstract}

Key words: frailty, Clinical Frailty Scale, ageing

\section{Why This Paper?}

This short paper is meant to be a guide for people new to the Clinical Frailty Scale. It should be seen as supplementing, not competing with, the many useful guides that have become available, especially in the setting of the COVID-19 pandemic. This work builds on existing resources about how to use the scale. Here we will discuss what frailty is, how the Clinical Frailty Scale arose, how it works now, and what can be done to check on its reliability and usefulness (both sit within the traditional notion of validity). We will introduce an updated version (CFS version 2.0) with revised level names and minor edits to level descriptions (Figure 1). We will also suggest some steps that may be helpful to those who wish to use frailty for assessment rather than for screening. These are meant to complement, not replace, their individual disciplinary approach. Finally, we will reflect on what we have learned about using the Clinical Frailty Scale over the years, and on the frailty discourse that began long before the Scale was developed. Most is grounded in evidence; that which is speculative we hope will be useful, if only for hypothesis generation.

The Clinical Frailty Scale is being used in many settings to help guide decisions that are based on the degree of frailty. That is neither rare nor, on its own, undesirable. Care plans should vary by the degree of frailty-for example, by the ability to climb stairs before being discharged, or to undertake (versus be assisted with) personal care. The COVID-19 pandemic brings with it an uncomfortable difference. With these assessments now come decisions about offering —or not offering - resources that are, or may become scarce, such as intubation or agents that increase blood pressure in someone in shock. Although rationing is not part of ordinary care, it is inevitable; if done properly, it can be made acceptable at the system level in the setting of a public health emergency. ${ }^{(1)}$

That being the case, many people less than familiar with the notion of frailty are now being called upon to conduct frailty assessments. This is challenging because the evaluation of people who are frail requires not only cognitive skills, but also the affective and attitudinal ones needed to work with people who have multiple, interacting medical and social problems. ${ }^{(2)}$

\section{What Is Frailty?}

Frailty is a state of increased risk. ${ }^{(3)}$ Inasmuch as most health risks increase with age, a handy way to think about frailty is that it is a risk compared with others of the same age.* Like the risk of dying, on average frailty increases with age. People are frail when they have more things wrong with them than do

\footnotetext{
*This conforms to the statistical notion of frailty as variability in risk compared with others with the same degree of exposure. The scale is built on the idea of age-related deficit accumulation. People of the same age are at greater risk in virtue of having more health deficits than do others of the same age. The Clinical Frailty Scale maps the consequences of this that precede death: decrements in high order functions.
} 


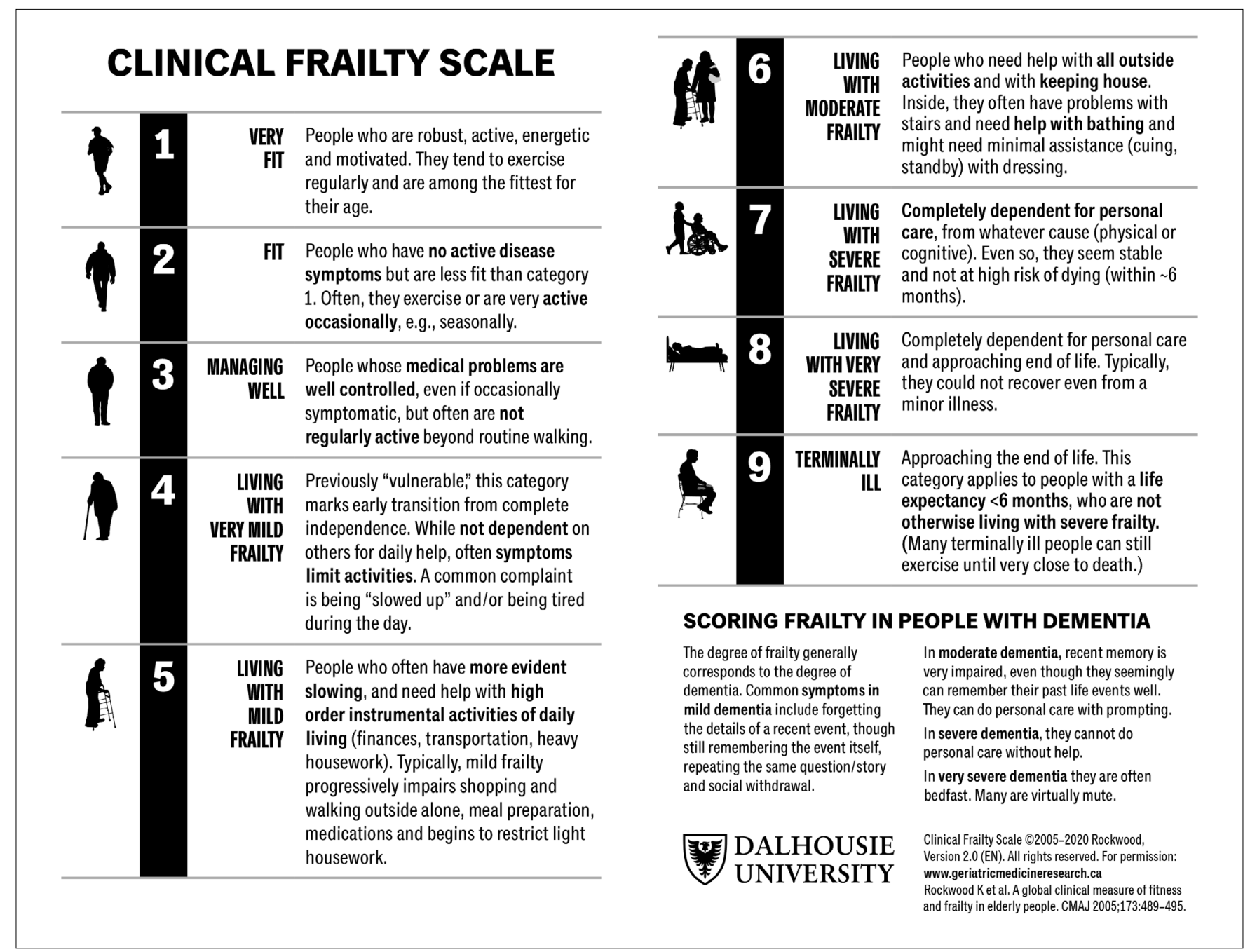

FIGURE 1. The Clinical Frailty Scale (CFS) version 2.0

others of the same age. Although that might not seem like a big deal, it is a real challenge for how we deliver health care. Medicine's tremendous success in treating people one illness at a time leads to the belief that is how people should get sick. As our population ages though, because frailty and age travel together, many of our patients have many things wrong at the same time. That is the challenge we face: their chance of having more things wrong with them increases as they age.

As these health deficits accumulate, they have clinical consequences, including in how diseases present, how often more than one illness is active at a time, how people are able to tell us about their symptoms or cooperate with our exams, how drugs work, and how likely it is that we can succeed similar to when treating people who have just a single predominant illness. By understanding frailty and changing what we offer to meet the needs of people who live with it, we can face that challenge. The Clinical Frailty Scale, developed in Halifax, Nova Scotia in the 1990s, considers the things that most often go wrong as age-related health deficits accumulate. (4) It robustly predicts adverse health outcomes in a variety of settings, including acute care. ${ }^{(5)}$

\section{What is the Clinical Frailty Scale?}

The Clinical Frailty Scale is an inclusive 9-point scale that was originally developed to summarize the overall level of fitness or frailty of an older adult after they had been evaluated by a health care professional. The scale is scored so that higher scores mean greater risk. It is not a questionnaire. Grading the degree of frailty requires clinical judgement that is based in part on screening criteria, and which then considers what broadly stratifies degrees of fitness and frailty. The scale focuses on items that can be readily observed without specialist training, including mobility, balance, use of walking aids, and the abilities to eat, dress, shop, cook, and bank. For this reason, scoring should match the description, and should not be based solely on the pictures that accompany each level.

The key to scoring the Clinical Frailty Scale is to determine the person's baseline health state. This is especially needed in clinical settings where health can change quickly. For example, many older patients in the Emergency Department who were fit two weeks ago (their baseline state), may appear to be frail while ill; a prospect that becomes more likely the longer they wait there. Still, as discussed below, 
their prognosis requires information about both their baseline degree of frailty, and the severity of their acute illness.

In ordinary time (i.e., with no pandemic), the Clinical Frailty Scale is used both in prognosis and to set care goals. In geriatric medicine, goals of care extend much beyond the often desultory exercise of "getting the code status". Usually much more relevant is understanding the baseline state and current decrements from it, which raise questions such as: "Does the patient have the manual dexterity to handle the mechanics of toileting? Do they need to climb stairs to get home?" If not, and depending on the capacity of whomever (if anyone) might be able to help them at discharge, goals of care need to be set in these domains. This information also facilitates prognosis: the greater the decline from the baseline state, the more energy it will take to recover. Given that intensity of effort may be limited by illness, this energy is often expressed as a function of time to recovery. Recovery time can be monitored from the start. It appears that the first 48 hours of hospitalization (preliminary data from Dr. Samuel D. Searle at our centre suggest up to 4 days in ICU patients) is information rich. ${ }^{(6)}$ Those who improve in how they move in bed do well; those who show decline fare especially poorly. In consequence, even recognizing the interplay between baseline frailty and severity of illness, many patients are well served by a trial of therapies. This provides a way to avoid the common, but false, dichotomy between "letting your father die" and "doing everything we can".

In a pandemic, these nuances may be less relevant when an older person has become ill enough that admission to the intensive care unit is being considered. Critical illness carries a clear age-related burden; in Italy, of the initial 18,366 COVID-19 deaths, $83 \%$ were aged 70 years and older. ${ }^{(7)}$ As in ordinary time, the stratification of risk must also consider the severity of illness. ${ }^{(8,9)}$ In critically ill people with COVID-19, this appears to be substantial for adults aged $65+$ years - the risk of death is more than $50 \%$ after 28 days. ${ }^{(10)}$

It is important to understand which older adults who are critically ill with COVID-19 disease might withstand both the illness and its treatment in an Intensive Care Unit. International responses vary, but seem to settle around a Clinical Frailty Scale score of $5^{(11)}$ to $6^{(12)}$ or higher being excluded from ICU admission.

Before reviewing how the scale is used, it is crucial to make a key additional point. The Clinical Frailty Scale, developed to study frailty in older adults, has largely not been validated in younger people. That is important because disability in younger people (including both acquired, as in spinal cord injury, and life-long, as in intellectual disability) does not have the same meaning for prognosis that it does with age-related disability. Understanding both this point and that the Clinical Frailty Scale is a judgement-based measure ${ }^{\dagger}$ of the baseline state, we can proceed.

tLike many skills in clinical medicine, judgement should increase with time. Particularly at the start, if how to classify the person is not clear, ask a colleague whose judgement you trust.
The scale can be introduced by saying something like: "I'd like to know how you are [your parent is] doing overall." We then ask about four features: how the person moved, functioned, thought, and felt about their health over the last two weeks. We can ask about which medications the person uses; experienced clinicians can quickly assay which illnesses are likely present from what medications are being prescribed and/or used. We also ask about how active the person is.

\section{Using the Clinical Frailty Scale to Grade Degrees of Fitness Prior to the Level of Risk Associated with Frailty}

How a person moves, functions, and think helps to delineate the first three levels of the scale. For example, consider a patient who is not impaired in any instrumental or personal activity of daily living (ADL), who is able to move readily, and who is taking an angio-converting enzyme (ACE) inhibitor. If that person is taking the ACE inhibitor for treatment of hypertension and exercises or engages in vigorous activity daily, their score would be Level 1- Very Fit. The same profile, with regular but less frequent or less vigorous physical activity would be scored as Level 2 - Fit (previously "Well"). Another person who fits the broad description and who is using the ACE inhibitor as part of post myocardial infarction management, but whose ischemic heart disease has been otherwise asymptomatic, would also be scored based on their degree of physical activity. If their symptoms were mostly controlled, but not entirely so, they would be scored as Level 3 - Managing Well. Likewise, a person on an ACE inhibitor as part of symptomatic management of congestive heart failure would be scored as Level 3 - Managing Well, as long as their symptoms did not limit activities, in which case they would be scored as Level 4 - Living with Very Mild Frailty (previously "Vulnerable").

\section{Using the Clinical Frailty Scale to Grade Clinically Meaningfully Increased Risk}

For Levels 4 to 7, mobility, function, and cognition are key factors. Each reflects high-order aspects of health: they integrate a lot of information. This means that there are many ways to have mobility problems, for example: a sprained ankle, diabetic nerve damage, dehydration, heart failure, kidney damage or pneumonia. In consequence, these key domains are sensitive signs of health, but are not very specific. It is the combination of impaired function and impaired mobility, which are commonly accompanied by several illnesses, that make it likely someone is frail.

Level 4 - previously "Vulnerable" is now Living with Very Mild Frailty, reflecting recent research with the Canadian Longitudinal Study of Aging that captures the increased risk with the corresponding degree of deficit accumulation. ${ }^{(13)}$ People with many chronic conditions often report incomplete symptom control, and of feeling "slowed up" or tired. A similar complaint is that their health stands in the way of doing as they wish, or that what they had done easily is now accomplished only with great effort. Otherwise, Level 4 is characterized by 
the person who, although not completely dependent in performing daily activities, seems at risk of at least mild dependence. Identifiable characteristics of this risk include incomplete symptom control and a reduction in demanding activities. Problems with heavy housework, lifting (e.g., difficulty taking out the trash) or climbing more than a flight of stairs are useful signs. Although these activities might still be attempted, often they are not done as well or as often. When asked, "compared to others of your own age, how would you rate your health?", many at this stage will no longer rate their health as "excellent", or even "good", but rather as "fair" or "poor".

Levels 5 to 7 relate to changes in function. Varying degrees of dependence in instrumental ADLs define Level 5 - Living with Mild Frailty (previously "Mildly Frail"). At this level, typically, there is no more pretense of doing heavy housework or the like-items that began to be impaired in Level 4. A person does not need to be dependent in all demanding activities to qualify as Level 4 , nor in all aspects of instrumental ADLs to qualify as Level 5 . We are interested in change; someone who never did the banking would not now be scored as dependent in that.

With Level 6 - Living with Moderate Frailty (previously "Moderately Frail"), dependence now extends past instrumental ADLs to intermediate ones, notably including dependence in bathing. Often at this level minimal assistance with personal care might be needed. Moderate dementia is the case when people who are dependent in their performance of instrumental ADLs can still do their basic or personal ADLs with prompting. This can also be the case in people who are cognitively intact, but whose disability obliges them to have someone nearby (so-called "standby assistance" or "set-up"). Again considering bathing, an example of moderate frailty might be requiring someone to draw the bath when the disabled person has difficulty managing the taps, or assisting with transferring in and out of the tub, or washing their hair. Notably, a systematic review found that bathing disability is a risk factor for greater disability in personal ADLs. ${ }^{(14)}$

Level 7 - Living with Severe Frailty (previously "Severely Frail") is characterized by progressive dependence in personal ADLs. People need not be dependent in every aspect of personal care to be scored as Level 7. When assessing functional dependence in intermediate (Level 6) and personal (Level 7) ADLs, lifelong habit is a less relevant consideration; most everyone needs to bathe, groom, and use the toilet. Still, people living with severe frailty can be mobile. Progressively taking to bed — but not being largely bedfast — is the hallmark of the progression of severe frailty.

\section{Using the Clinical Frailty Scale in People Towards the End of Life}

The understanding of what happens at the end of life has evolved in relation to its association with ageing. Older people who are terminally ill are much more likely to receive formal palliative care if they have a diagnosis of cancer than if they have a disease with a recognized terminal phase, such as dementia or heart failure. ${ }^{(15)}$
Level 8 - Living with Very Severe Frailty (previously "Very Severely Frail") is the not uncommon state in which a frail person takes to bed, often for weeks, prior to dying. This is either heralded by an identifiable episode, such as an infection, or the person just slips away, commonly after some days of reduced oral intake. Very severely frail people who die without a single apparent cause typically follow such a trajectory, commonly without much pain or even distress, often, with the exception of impaired bowel function.

Level 9 - Terminally III is notable for being the only level in which the current state trumps the baseline state, in that the terminally ill person might have been operating at any frailty level at baseline. On the Clinical Frailty Scale card, this person is pictured seated in a chair. This reflects the fact that many older adults who are dying with a single system illness - notably cancer-have a reasonable level of function until about the very end. That is why we portray the situation in that way. Even so, if a terminally ill person was completely dependent for personal care at baseline, they would be scored as Level 8.

\section{Final Hints About Scoring and Next Steps}

Within each level of the Clinical Frailty Scale, individual characteristics will vary. About $80 \%$ or more of people will fit the description offered for a given level. If they fit two categories equally well, in routine care it is best to score the scale at the higher or more dependent level. Sometimes we see people who are dependent in a single instrumental ADL that arises in a specific circumstance (e.g., relying on someone whom they trust to do banking duties due to the closure of a nearby bank branch or difficulty with the automated banking). In that case, the determination will often rest on the extent to which the person is aware of income and outflow; being aware of it and knowing that it remains a matter of importance can suffice. We recognize that there is likely to be some variability in judgement in these circumstances, especially in the extent to which the rater or the person (or the informant) feel comfortable discussing such matters. This is inherent in a judgement-based measure and, in our view, a price worth paying compared with attempting to automate scoring that can specify all the variants its designers can imagine.

\section{Scoring the Clinical Frailty Scale in People with Cognitive Impairment}

The degree of dementia generally corresponds to the degree of frailty. That is, mild dementia would go with mild frailty. In both cases, the person is independent in their personal or basic ADLs, but dependent in one or more instrumental ADLs. These accompany common symptoms of mild dementia: being able to recall a recent event but forgetting its details, repeating questions throughout the day, and no longer being able to use routines or cues to aid in either not losing items or finding them once lost.

In moderate dementia, recent memory is very impaired, even though they seemingly can remember their past life events well. As with moderate frailty, they can do their 
personal care with prompting or set-up. In severe dementia, as in severe frailty, people have progressive difficulty in performing personal ADLs and require increasing amounts of hands-on assistance.

\section{Using a Classification Tree to Improve the Reliability of Scoring}

As of Spring 2020, we are beginning a multi-centre, multicountry study using a classification tree to assist with grading the degree of frailty; clinical judgement is still required to score the Clinical Frailty Scale. Our goal is to improve the reliability of scoring the CFS using the classification tree. Inasmuch as the reliability will be measured in relation to the score given by an experienced rater, this is also a form of validity. We will further test validity by the ability to predict several outcomes in relation to COVID-19, including progression from mild to severe frailty, critical illness, mortality, and health care use.

\section{Summary}

This is a brief account of key points in using the Clinical Frailty Scale. It can be read in conjunction with two contemporary papers in the Canadian Geriatrics Society CME Journal $^{(16,17)}$ and with a paper on the Pictorial Fit-Frail Scale. ${ }^{(18)}$ The latter can help to bridge between screening and assessment measures, and can itself be summarized by the Clinical Frailty Scale. Additional resources include "Top Tips to help you use the Clinical Frailty Scale" developed by our group, ${ }^{(19)}$ a Clinical Frailty Scale App developed by the Acute Frailty Network in the $\mathrm{UK}^{(20)}$ (this may be a useful alternative to the classification tree we are testing), a Clinical Frailty Scale Training Module developed by the AIMS Research Group at the Ottawa Hospital, ${ }^{(21)}$ and a Clinical Frailty Scale Education Tool developed by the Non-Consultant Hospital Doctors of the Cork University Hospital. ${ }^{(22)}$

Our target audience is people who are new to the Clinical Frailty Scale and those who may be teaching about its use. We put this work in the context that it must be used carefully and combined with clinical judgement. ${ }^{(23)}$ It should not be used in younger people in whom lifelong or single system disability does not have the same prognostic value as does that seen as a manifestation of wide-spread deficit accumulation. Even so, applied properly, the Clinical Frailty Scale seems likely to be much preferable to age when decisions must be made about allocating scarce resources. ${ }^{(17,24)}$ An additional point to underscore is this: grading the degree of frailty in the context of COVID-19 is not the same as using it in ordinary care in geriatric medicine. In geriatric medicine, the goal of grading the degree of frailty is both to ease communication through a shared language, and also as the foundation for a care plan. The care plan needs to address the gap between the current level of function, cognition, mobility, and social relationships (especially with the primary carer) and how those have changed with the acute illness (when present). There the focus is on trying to restore the baseline state, or as close to that as might be achieved. This is a crucial distinction, and one that requires considerable skill to define, negotiate, evaluate, and adjust over the course of an inpatient stay. It should not be confused with the more instrumental purpose to which the Clinical Frailty Scale is now being put, which is no substitute for a patient-centred care plan. Even so, we hope that widespread use of the Clinical Frailty Scale can at least introduce new users to the manifold benefits that are possible with such an approach.

We have emphasized key general points: assay the baseline state, do not use it with younger people or those with stable single-system disabilities, use judgement, and be prepared to examine your judgement as experience grows. Several specific points relating to each level of the scale are noted. Finally, we draw to attention that this is an inclusive scale. There is room on it for everyone - a reminder of the frailty that we all face, if we are lucky enough to live a long life.

\section{CONFLICT OF INTEREST DISCLOSURES}

Kenneth Rockwood has asserted copyright of the Clinical Frailty Scale through Dalhousie University. Use is free for research, education, or not-for-profit care. (Users are asked not to change it or charge for its use.) In addition to academic and hospital appointments, he is President and Chief Science Officer of DGI Clinical, which in the last five years has contracts with pharma and device manufacturers (Baxter, Baxalta, Biogen, Shire, Hollister, Nutricia, Roche, Otsuka) on individualized outcome measurement. In 2017 he attended an advisory board meeting with Lundbeck. He is Associate Director of the Canadian Consortium on Neurodegeneration in Aging, which is funded by the Canadian Institutes of Health Research (CAN-137794), with additional funding from the Alzheimer Society of Canada and several other charities. He receives research support through grants from the Canadian Institutes of Health Research, the Canadian Frailty Network, the Nova Scotia Health Research Foundation, the Nova Scotia Health Authority Research Fund, the Dalhousie Medical Research Fund as the Kathryn Allen Weldon Professor of Alzheimer Research, and the Fountain Family Innovation Fund of the QEII Health Science Centre Foundation.

Olga Theou declares that no conflicts of interest exist.

\section{REFERENCES}

1. World Health Organization. Ethical considerations in developing a public health response to pandemic influenza. Geneva, Switzerland:WHO; 2007. https://www.who.int/csr/resources/publications/ WHO_CDS_EPR_GIP_2007_2c.pdf Accessed May 32020.

2. Rockwood K. Medical management of frailty: confessions of a gnostic. CMAJ. 1997;157(8):1081-84.

3. Rockwood K, Howlett SE. Age-related deficit accumulation and the diseases of ageing. Mech Ageing Dev. 2019;180:107-16.

4. Rockwood K, Song X, MacKnight C, et al. A global clinical measure of fitness and frailty in elderly people. CMAJ. 2005;173(5):489-95.

5. Theou O, Squires E, Mallery K, et al. What do we know about frailty in the acute care setting? A scoping review. BMC Geriatr. 2018;18(1):139. 


\section{ROCKWOOD \& THEOU: ALLOCATING SCARCE HC RESOURCES}

6. Hatheway OL, Mitnitski A, Rockwood K. Frailty affects the initial treatment response and time to recovery of mobility in acutely ill older adults admitted to hospital. Age Ageing. 2017; 46(6):920-25.

7. Boccardi V, Ruggiero C, Mecocci P. COVID-19: a geriatric emergency. Geriatrics. (Basel). 2020;5(2):E24.

8. Romero-Ortuno R, Wallis S, Biram R, et al. Clinical frailty adds to acute illness severity in predicting mortality in hospitalized older adults: an observational study. Eur J Intern Med. 2016;35:24-34.

9. Pulok M, Theou O, van der valk A, et al. The role of illness acuity on the association between frailty and mortality in Emergency Department patients referred to internal medicine. Age Ageing 2020 May 11:afaa089. Published online ahead of print. Correction in Age Ageing $2020 \mathrm{Jul}$ 24:afaa153. Published online ahead of print.

10. Bhatraju PK, Ghassemieh BJ, Nichols M, et al. Covid-19 in critically ill patients in the Seattle region-case series. $N$ Engl JMed.2020;NEJMoa2004500. Published online ahead of print, 2020 Mar 30.

11. National Institute for Health and Care Excellence. COVID-19 rapid guidelines: critical acre in adults (update $27 \mathrm{March} 2020$ ). London, UK: NICE; 2020. https://www.nice.org.uk/guidance/ng159/ resources/critical-care-admission-algorithm-pdf-8708948893

12. The Swiss Society of Emergency and Rescue Medicine. COVID-19: Prehospital triage and care under resource scarcity in the hospital sector. V2.0-EN / 06.04.2020. Geneva, Switzerland: SSERM; 2020. https://www.sgnor.ch/fileadmin/ user_upload/Dokumente/Algo_Covid_enV2c.pdf

13. Perez-Zepeda MU, Godin J, Armstrong JJ, et al. Frailty among middle-aged and older Canadians: Population norms for the Frailty Index using the Canadian Longitudinal Study on Aging. and Ageing (in press)

14. Golding-Day M, Whitehead $\mathrm{P}$, Radford $\mathrm{K}$, et al. Interventions to reduce dependency in bathing in community dwelling older adults: a systematic review. Syst Rev. 2017;6(1):198.

15. Hill AD, Stukel TA, Fu L, et al. Trends in site of death and health care utilization at the end of life: a population-based cohort study. CMAJ Open. 2019;7(2):E306-E315.
16. Varshney N. COVID-19 and ethical critical care triage - utilizing this period to further knowledge translation of frailty and ICU evidence during a pandemic and beyond. Can Geriatr Soc CME J. 2020;10(1). Available from: https://canadiangeriatrics. ca/wp-content/uploads/2020/05/Varshney_Covid19-Formatted_ FINAL-1.pdf

17. Rockwood K, Dykes L. Communicating an ethical basis for decision-making in relation to frailty and allocation of scarce resources in the COVID-19 pandemic. Can Geriatr Soc CMEJ. 2020;10(1). https://canadiangeriatrics.ca/wp-content/uploads/ 2020/05/Communicating-an-Ethical-Basis-for-Decision-Making -COVID-19.pdf

18. Theou O, Andrew M, Ahip SS, et al. The Pictorial Fit-Frail Scale: developing a visual scale to assess frailty. Can Geriatr J. 2019;22;(2):64-74.

19. Rockwood K, Theou O, Fay S, et al. Top Tips to help you use the Clinical Frailty Scale. Accessed on July 28, 2020. Available from: www.dal.ca/sites/gmr/our-tools/clinical-frailty-scale/ cfs-guidance.html

20. Acute Frailty Network. Clinical Frailty Scale App. Accessed on July 28, 2020. Available from: www.acutefrailtynetwork. org.uk/Clinical-Frailty-Scale/Clinical-Frailty-Scale-App

21. AIMS Research Group. Clinical Frailty Scale Training Module. Accessed on July 28, 2020. Available from: https://rise.articulate. com/share/deb4rT02lvONbq4AfcMNRUudcd6QMts3\#/

22. Moloney E, Ahern E, O'Caoimh R. Clinical Frailty Scale Education Tool. Accessed on July 28, 2020. Available from: https:/ucc.cloud.panopto.eu/Panopto/Pages/Viewer. aspx?id=ce9a4d94-fc1c-44ca-8a39-ab9600f624f3

23. Hubbard RE, Maier AB, Hilmer SN, et al. Frailty in the face of COVID-19. Age Ageing. 2020 Jul 1;49(4):499-500.

24. Montero-Odasso, Hogan DB, Lam R. et al. Age alone is not adequate to determine health-care resource allocation during the COVID-19 pandemic. Can Geriatr J. 2020;23:152-54.

Correspondence to: Kenneth Rockwood, MD, Suite 1421 Veterans Memorial Bldg., 5955 Veterans Memorial Lane, Halifax, NS B3H 2E1

E-mail: kenneth.rockwood@dal.ca 